\title{
ON AN ELASTIC DISSIPATION MODEL AS CONTINUOUS APPROXIMATION FOR DISCRETE MEDIA
}

\author{
I. V. ANDRIANOV, J. AWREJCEWICZ, AND A. O. IVANKOV \\ Received 18 August 2006; Accepted 13 September 2006
}

Construction of an accurate continuous model for discrete media is an important topic in various fields of science. We deal with a $1 \mathrm{D}$ differential-difference equation governing the behavior of an $n$-mass oscillator with linear relaxation. It is known that a stringtype approximation is justified for low part of frequency spectra of a continuous model, but for free and forced vibrations a solution of discrete and continuous models can be quite different. A difference operator makes analysis difficult due to its nonlocal form. Approximate equations can be obtained by replacing the difference operators via a local derivative operator. Although application of a model with derivative of more than second order improves the continuous model, a higher order of approximated differential equation seriously complicates a solution of continuous problem. It is known that accuracy of the approximation can dramatically increase using Padé approximations. In this paper, one- and two-point Padé approximations suitable for justify choice of structural damping models are used.

Copyright (c) 2006 I. V. Andrianov et al. This is an open access article distributed under the Creative Commons Attribution License, which permits unrestricted use, distribution, and reproduction in any medium, provided the original work is properly cited.

\section{Introduction}

Many different phenomenological theories are used to describe energy dissipation in vibrating elastic bodies [5]. In $[6,11,12]$ a new dissipation model for beams with damping assumed to be proportional to the beam moment has been proposed. Namely, the following equation has been studied:

$$
\rho y_{t t}(x, t)-\mu y_{t_{x x}}(x, t)+E I y_{x x x x}(x, t)=0,
$$

where $\rho$ is the density of the beam material, $\mu$ is the damping parameter, $E$ is the Young coefficients, and $I$ is the first moment of the beam cross-section. 
2 On the elastic dissipation model

On the other hand, in $[6,11]$ the models studied are of the form

$$
y_{t t}(x, t)+B y_{t}(x, t)+A y(x, t)=0,
$$

where $A$ is the relevant elasticity operator.

Notice that the operator $B$ is related in various ways to the positive square root of $A$.

In [12] spatial hysteresis model has been reviewed and then shear diffusion model has been introduced. The last one has been obtained by coupling the originally conservative elastic equations to two different diffusion processes. Then decoupling process has been used to project the coupled system onto the subspace corresponding to the lateral displacements. It has been shown that the projected system agrees in many significant aspects with the spatial hysteresis model.

From a mathematical standpoint, this model has been also analyzed in $[13,15]$.

We will analyze the applicability of (1.1) by using discrete media with linear relaxation and its continuous approximation.

\section{String model}

We deal with an $n$-mass damped oscillator. The governing equations of motion follow

$$
\begin{gathered}
m y_{j t t}+\alpha y_{j t}=c D\left(y_{j}\right)+s_{j}(t), \quad j=1, \ldots, n, \\
y_{0}=y_{n+1}=0
\end{gathered}
$$

where $D\left(y_{i}\right)=y_{j+1}-2 y_{j}+y_{j-1}, y_{j}(t)$ is the displacement of the $j$ th point, $s_{j}(t)$ is the external force acting on $j$ th point, $\alpha$ is the coefficient of linear relaxation, $m$ is the mass, $c$ is the rigidity, and index " $t$ " (e.g., $\left.y_{j t}(t)\right)$ denotes differentiation with respect to $t$.

The following initial conditions are applied:

$$
y_{j}(t)=y_{j t}(t)=0 \quad \text { for } t=0 .
$$

Usually for large values of $n$, the string-like continuous approximation to the above discrete problem is applied:

$$
\begin{gathered}
m y_{t t}(x, t)+\alpha y_{t}(x, t)=\operatorname{ch}^{2} y_{x x}(x, t)+s(x, t), \\
y(0, t)=0, \quad y(l, t)=0 \\
y(x, 0)=y_{t}(x, 0)=0
\end{gathered}
$$

where $l=(n+1) h$.

The function $s(x, t)$ represents a continuous approximation of the discretely defined function $s_{j}(t)$. Observe that it is defined with an accuracy to any arbitrary function which equals zero in the nodal points $x=j h, j=1,2, \ldots, n$. For this reason, from a set of interpolating functions one may choose, say, the smoothest function owing to filtration of fast oscillating terms. This problem has been solved by Kunin [8], who proposed the following approximation:

$$
s(x, t)=\sum_{k=1}^{n} s_{k}(t) \frac{\sin (k \pi x / h)}{\pi k h} .
$$


The following relation between discrete and continuous systems holds:

$$
y_{j}(t)=y(j h, t), \quad j=1, \ldots, n \text {. }
$$

Note that system (2.1) can be reduced to one pseudodifferential equation. For this purpose the translation operator $\exp ( \pm \partial / \partial n)$ is introduced, and the following formal identity holds: $\exp ( \pm \partial / \partial n) y_{i}=y_{i \pm 1}$. Then (see [14] for more details)

$$
D y_{i}=\left[\exp \left(\frac{\partial}{\partial n}\right)-2+\exp \left(-\frac{\partial}{\partial n}\right)\right] y_{i}=4 \sin ^{2}\left(-\frac{i h}{2} \frac{\partial}{\partial n}\right) y_{i}
$$

It means that difference operator $D$ in the right-hand side of (2.1) can be represented by the following pseudodifferential operator:

$$
D=\sin ^{2}\left(-\frac{i h}{2} \frac{\partial}{\partial x}\right)
$$

The following McLaurin series is applied:

$$
\sin ^{2}\left(-\frac{i h}{2} \frac{\partial}{\partial x}\right)=-\left(\frac{h^{2}}{4} \frac{\partial^{2}}{\partial x^{2}}+\frac{h^{4}}{48} \frac{\partial^{4}}{\partial x^{4}}+\frac{h^{6}}{1440} \frac{\partial^{6}}{\partial x^{6}}+\cdots\right)
$$

Taking into account the first term in the series (2.11), the string-like continuous approximation (2.4) is obtained. Taking into account three first terms, the following higherorder approximation is found:

$$
m \frac{\partial^{2} y}{\partial t^{2}}+\alpha \frac{\partial y}{\partial t}=\operatorname{ch}^{2}\left(\frac{\partial^{2} y}{\partial x^{2}}+\frac{h^{4}}{12} \frac{\partial^{4} y}{\partial x^{4}}+\frac{h^{6}}{360} \frac{\partial^{6} y}{\partial x^{6}}\right)+s(x, t)
$$

Initial conditions have the form (2.6).

The problem associated with the boundary conditions requires a more subtle analysis. Note that the boundary conditions for an $n$-mass oscillator (2.2) are transited automatically into boundary conditions for the string (2.5). However, if a continuous approximation has a relatively high order (see (2.12)), one has to define $y_{k}(t)$ for both $k<0$ and for $k>n+1$. If one supposes periodic chain, then

$$
\begin{gathered}
y_{-1}(t)=-y_{1}(t), y_{-2}(t)=-y_{2}(t), \text { and so forth, } \\
y_{n+2}(t)=-y_{n}(t), y_{n+3}(t)=-y_{n-1}(t), \text { and so forth, }
\end{gathered}
$$

and the following boundary conditions associated with (2.12) are then obtained:

$$
y=y_{x x}=y_{x x x x}=0 \quad \text { for } x=0, l .
$$

Initial conditions have the form (2.6).

Previously, we used the expansion of a pseudodifferential operator into the McLaurin series. However, more effective results may be obtained by using Padé approximations (PA). In $[7,9,10]$ the continuous models are constructed using one-point PA. A brief 
4 On the elastic dissipation model

description of the PA follows. Let the function $F(\varepsilon)$ is represented by the McLaurin series

$$
F(\varepsilon)=\sum_{i=0}^{\infty} a_{i} \varepsilon^{i} \quad \text { for } \varepsilon \longrightarrow 0 .
$$

The PA $[m / n]$ is defined through the fractional rational function

$$
F_{[m / n]}(\varepsilon)=\frac{\sum_{i=0}^{m} \beta_{i} \varepsilon^{i}}{1+\sum_{i=1}^{n} \gamma_{i} \varepsilon^{i}},
$$

whose first $m+1$ coefficients of the associated McLaurin series for $F_{[m / n]}(\varepsilon)$ overlap with the first terms of the McLaurin series for $F(\varepsilon)$.

Let us take into account only two terms of the expansion (2.11). Then one has the following continuous model:

$$
\begin{gathered}
m \frac{\partial^{2} y}{\partial t^{2}}+\alpha \frac{\partial y}{\partial t}=c^{2}\left(\frac{\partial^{2} y}{\partial x^{2}}+\frac{h^{4}}{12} \frac{\partial^{4} y}{\partial x^{4}}\right)+s(x, t), \\
y=y_{x x}=0 \quad \text { for } x=0, l
\end{gathered}
$$

Initial conditions have the form (2.6).

The PA of the truncated series (2.11), when one takes into account only two terms of expansion, has the form

$$
\frac{\partial^{2} / \partial x^{2}}{1-\left(h^{2} / 12\right)\left(\partial^{2} / \partial x^{2}\right)} .
$$

It leads to the following continuous model:

$$
\left(1-\frac{h^{2}}{12} \frac{\partial^{2}}{\partial x^{2}}\right)\left(m y_{t t}+\alpha y_{t}\right)-c h^{2} y_{x x}=c\left(1-\frac{h^{2}}{12} \frac{\partial^{2}}{\partial x^{2}}\right) s(x, t)
$$

with the boundary conditions (2.5) and the initial conditions (2.6).

According to references [1-4], it is clear that application of two-point PA (TPPA) is more efficient than the one-point PA. Recall briefly the definition of TPPA. Let

$$
\begin{aligned}
& F(\varepsilon)=\sum_{i=0}^{\infty} a_{i} \varepsilon^{i} \quad \text { for } \varepsilon \longrightarrow 0, \\
& F(\varepsilon)=\sum_{i=0}^{\infty} b_{i} \varepsilon^{i} \quad \text { for } \varepsilon \longrightarrow A .
\end{aligned}
$$

TPPA $F_{p}$ is represented by the following fractional rational function: $F_{p}=\sum_{i=0}^{m} \beta_{i} \varepsilon^{i} /$ $\left(1+\sum_{i=1}^{n} \gamma_{i} \varepsilon^{i}\right)$, whose first $k$ coefficients of the associated McLaurin series and $m+n+$ $1-k$ first coefficients of its development into the Taylor series in the point $x=A$ overlap with the first coefficients of the series (2.21) and (2.22).

In order to construct TPPA, two limiting solutions are required. Let us analyse expression

$$
D(y)=y_{j+1}-2 y_{j}+y_{j-1} \text {. }
$$


In one limiting case (long-wave oscillations) $D(y)$ may be approximated as $D(y) \approx y_{x x}$. In the second limiting case (saw-tooth oscillations [1-4]) one obtains $y_{j+1}=-y_{j}$, and then $D(y)=-4 y_{j}$. Assuming $s_{j}(t)=0$, a frequency of vibrations of the discrete system $\omega$ can be found from the following equation:

$$
m \omega^{2}+\alpha \omega+4=0 .
$$

The equation of continual approximation is now cast in the form

$$
\left(1-h^{2} \delta^{2} \frac{\partial^{2}}{\partial x^{2}}\right)\left(m y_{t t}+\alpha y_{t}\right)-c h^{2} y_{x x}=0
$$

where the parameter $\delta^{2}$ should be determined.

A solution to $(2.25)$, taking into account the wave length $h$ regarding longitudinal coordinate has the form $y=A \exp (i \omega t) \sin (\pi x / h)$, where $\omega$ is defined by the following equation:

$$
m \omega^{2}+\alpha \omega+\frac{\pi^{2}}{1+\delta^{2} \pi^{2}}=0
$$

Comparison of (2.24) and (2.26) yields the estimation $\delta^{2}=1 / 4-\pi^{-2}$.

Finally, the modified continuous approximation may be written as follows:

$$
\left(1-\delta^{2} h^{2} \frac{\partial^{2}}{\partial x^{2}}\right)\left(m y_{t t}+\alpha y_{t}\right)-c h^{2} y_{x x}=\left(1-\delta^{2} h^{2} \frac{\partial^{2}}{\partial x^{2}}\right) s(x, t)
$$

Attached boundary and initial conditions have the form of (2.5) and of (2.6), respectively.

\section{Beam model}

In what follows the transversal vibrations of the $n$-mass chain are analysed.

The governing equations read

$$
m \frac{\partial^{2} y_{i}}{\partial t^{2}}+\alpha \frac{\partial y_{i}}{\partial t}+c\left(6 y_{i}-4 y_{i+1}-4 y_{i-1}+y_{i+2}+y_{i-2}\right)=s_{i}(t), \quad i=1,2, \ldots, n,
$$

with boundary conditions

$$
y_{0}=y_{n+1}=0, \quad y_{-1}=-y_{1}, \quad y_{n+2}=-y_{n},
$$

and initial conditions (2.3).

A typical continuous approximation of the system (3.1) has the form

$$
m \frac{\partial^{2} y(x, t)}{\partial t^{2}}+\alpha \frac{\partial y(x, t)}{\partial t}+c_{1} \frac{\partial^{4} y}{\partial x^{4}}=s(x, t), \quad c_{1}=c h^{4}
$$


6 On the elastic dissipation model

with the attached

$$
y=y_{x x}=0 \quad \text { for } x=0, l
$$

and initial (2.6) conditions.

The obtained continuous approximation can be improved. For this purpose, one may formally replace the difference operator in (3.1) via the pseudodifferential operator in the following way:

$$
m \frac{\partial^{2} y}{\partial t^{2}}+\alpha \frac{\partial y}{\partial t}+16 c \sin ^{4}\left(-\frac{i h}{2} \frac{\partial}{\partial x}\right) y=0
$$

The pseudodifferential operator can be developed into the McLaurin series of the form

$$
16 \sin ^{4}\left(-\frac{i h}{2} \frac{\partial}{\partial x}\right)=\frac{h^{4} \partial^{4}}{\partial x^{4}}+\frac{h^{6}}{6} \frac{\partial^{6}}{\partial x^{6}}+\frac{h^{8}}{80} \frac{\partial^{8}}{\partial x^{8}}+\ldots
$$

Taking into account only the first term in expression (3.6), one obtains the classical continuous approximation (3.3). However, taking into account the first three terms, the obtained approximation reads

$$
m \frac{\partial^{2} y}{\partial t^{2}}+\alpha \frac{\partial y}{\partial t}=c_{1} \frac{\partial^{4} y}{\partial x^{4}}\left(1+\frac{h^{2}}{6} \frac{\partial^{2}}{\partial x^{2}}+\frac{h^{4}}{80} \frac{\partial^{4}}{\partial x^{4}}\right) y+s(x, t)
$$

with boundary conditions (obtained using (2.13))

$$
y=y_{x x}=y_{x x x x}=y_{x x x x x x}=0 \text { for } x=0, l
$$

and initial conditions (2.6).

A transformation of the first two terms of the series (3.6) into the PA gives the following result:

$$
h^{4} \frac{\partial^{4}}{\partial x^{4}}\left(1+\frac{h^{2}}{6} \frac{\partial^{2}}{\partial x^{2}}\right) \sim h^{4} \frac{\partial^{4}}{\partial x^{4}} \frac{1}{1-\left(h^{2} / 6\right)\left(\partial^{2} / \partial x^{2}\right)} .
$$

Hence, a continuous approximation reads

$$
c_{1} \frac{\partial^{4} y}{\partial x^{4}}+\left(1-\frac{h^{2}}{6} \frac{\partial^{2}}{\partial x^{2}}\right)\left(m \frac{\partial^{2} y}{\partial t^{2}}+\alpha \frac{\partial y}{\partial t}-s(x, t)\right)=0,
$$

and the boundary conditions are defined by (3.4), whereas initial conditions are governed by (2.6).

TPPA is constructed using two limiting cases for expression

$$
D_{1}(y)=\left(6 y_{i}-4 y_{i+1}-4 y_{i-1}+y_{i+2}+y_{i-2}\right)
$$


For long-wave vibrations one obtains $D_{1}(y) \approx y_{x x x x}$, whereas for saw-tooth vibrations $\left(y_{i+1}=-y_{i}\right)$ one gets $D(y)=16 y_{i}$. Now we will construct continuous approximation

$$
c_{1} \frac{\partial^{4} y}{d x^{4}}+\left(1-\beta^{2} h^{2} \frac{\partial^{2}}{\partial x^{2}}\right)\left(m \frac{\partial^{2} y}{d t^{2}}+\alpha \frac{\partial y}{\partial t}-s(x, t)\right)=0
$$

and we will choose the parameter $\beta^{2}$.

Applying the so far described approach for the string model, one obtains $\beta^{2}=$ $1 / 16-\pi^{-4}$.

The boundary conditions for (3.12) have the form of (3.4).

\section{Conclusions}

As the authors of $[13,15]$ show, the proposed model in $[6,11,12]$ of energy dissipations (1.1) posses many disadvantages. In this work, we show that starting with a discrete model with linear relaxation is impossible to obtain directly model (1.1). Our consideration indicates also the validity of the following conclusion. Namely, application of one of the discussed dissipation models can not be directly motivated by mathematical analysis. It is rather necessary to carry out an experimental investigation to make the final choice.

\section{Acknowledgments}

Authors are thankful to W. T. van Horssen for the valuable comments and suggestions and anonymous reviewers for important critical remarks.

\section{References}

[1] I. V. Andrianov, Continuous approximation of higher-frequency oscillation of a chain, Doklady Akademii Nauk Ukrainskoj SSR, Seriya A 2 (1991), 13-15 (Russian).

[2] I. V. Andrianov and J. Awrejcewicz, On the average continuous representation of an elastic discrete medium, Journal of Sound and Vibration 264 (2003), no. 5, 1187-1194.

[3] _ Continuous models for 1D discrete media valid for higher-frequency domain, Physics Letters A 345 (2005), no. 1-3, 55-62.

[4] Continuous models for chain of inertially linked masses, European Journal of Mechanics A/Solids 24 (2005), no. 3, 532-536.

[5] C. W. Bert, Material damping: an introductory review of mathematical models, measures and experimental technique, Journal of Sound and Vibration 29 (1973), no. 2, 129-153.

[6] G. Chen and D. L. Russell, A mathematical model for linear elastic systems with structural damping, Quarterly of Applied Mathematics 39 (1982), no. 4, 433-454.

[7] M. A. Collins, A quasicontinuum approximation for solitons in an atomic chain, Chemical Physics Letters 77 (1981), no. 2, 342-347.

[8] I. A. Kunin, Elastic Media with Microstructure. I. One-Dimensional Models, Springer Series in Solid-State Sciences, vol. 26, Springer, Berlin, 1982.

[9] Ph. Rosenau, Dynamics of nonlinear mass-spring chains near the continuum limit, Physics Letters A 118 (1986), no. 5, 222-227.

[10] Hamiltonian dynamics of dense chains and lattices: or how to correct the continuum, Physics Letters A 311 (2003), no. 1, 39-52.

[11] D. L. Russell, On the positive square root of the fourth derivative operator, Quarterly of Applied Mathematics 46 (1988), no. 4, 751-773. 
[12] _ A comparison of certain elastic dissipation mechanisms via decoupling and projection techniques, Quarterly of Applied Mathematics 49 (1991), no. 2, 373-396.

[13] W. T. van Horssen and M. A. Zarubinskaya, On an elastic dissipation model for a cantilevered beam, Quarterly of Applied Mathematics 61 (2003), no. 3, 565-573.

[14] J. A. D. Wattis, Quasi-continuum approximations to lattice equations arising from the discrete nonlinear telegraph equation, Journal of Physics A: Mathematical and General 33 (2000), no. 33, 5925-5944.

[15] M. A. Zarubinskaya and W. T. van Horssen, On an improved elastic dissipation model for a cantilevered beam, Quarterly of Applied Mathematics 63 (2005), no. 4, 681-690.

I. V. Andrianov: Institute of General Mechanics, RWTH Aachen University,

Templergraben 64, 52062 Aachen, Germany

E-mail address: igor_andrianov@inbox.ru

J. Awrejcewicz: Department of Automatics and Biomechanics, Technical University of Łód;

Stefanowski Street 1/15, 90-924 Łód, Poland

E-mail address: k16@p.lodz.pl

A. O. Ivankov: Prydniprovska State Academy of Civil Engineering and Architecture, Chernishevskogo Street 24 a, 49005 Dnepropetrovsk, Ukraine

E-mail address: igor_andrianov@hotmail.com 


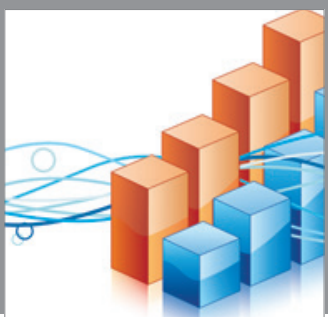

Advances in

Operations Research

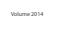

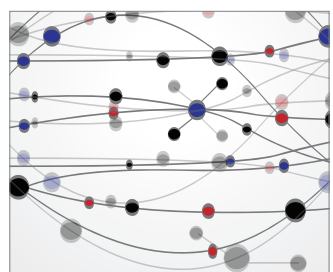

\section{The Scientific} World Journal
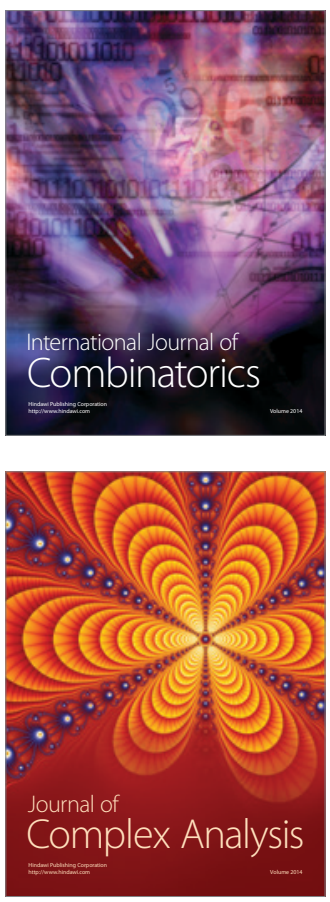

International Journal of

Mathematics and

Mathematical

Sciences
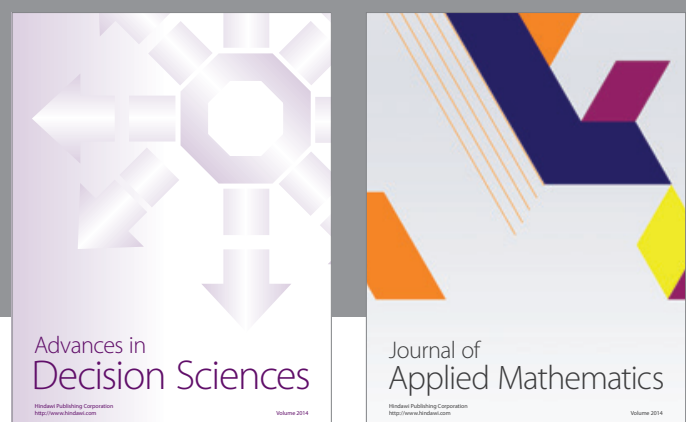

Journal of

Applied Mathematics
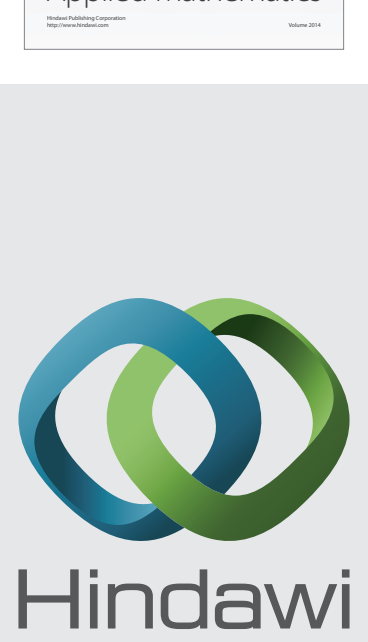

Submit your manuscripts at http://www.hindawi.com
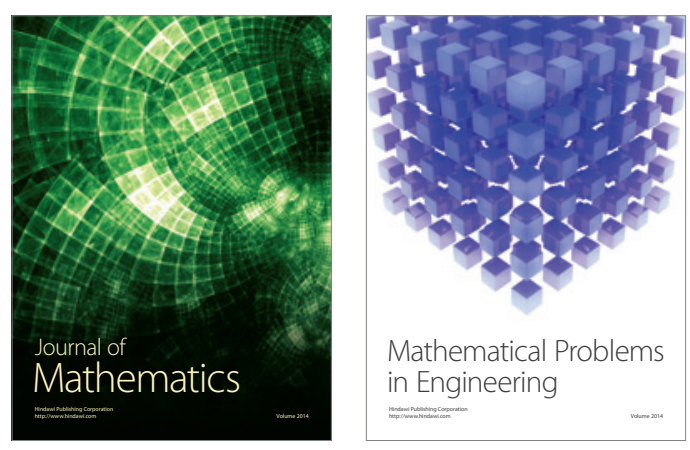

Mathematical Problems in Engineering
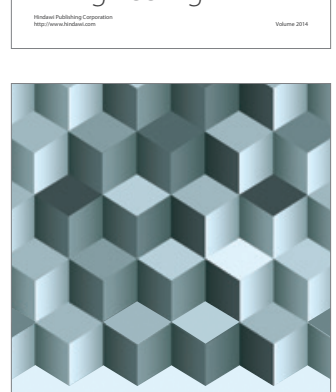

Journal of

Function Spaces
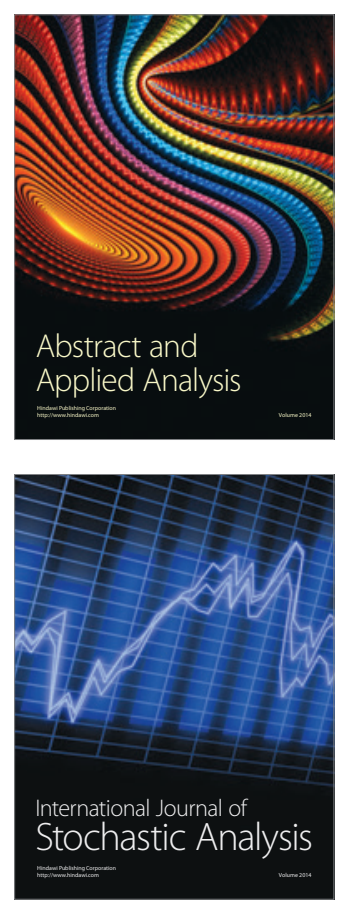

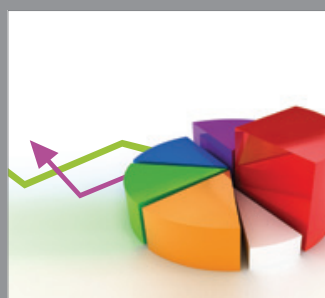

ournal of

Probability and Statistics

Promensencen
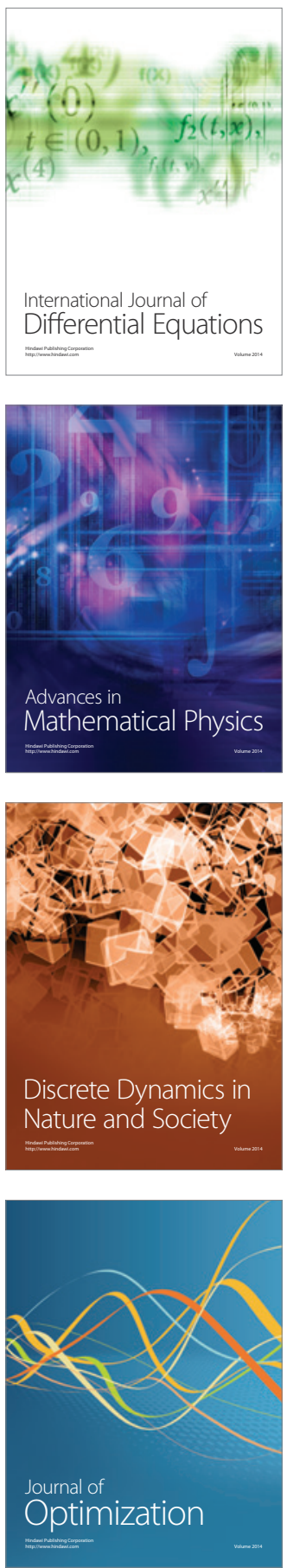